\title{
LA EVOLUCIÓN DE LA RESPONSABILIDAD SOCIAL EMPRESARIAL A TRAVÉS DE LAS TEORÍAS ORGANIZACIONALES
}

\section{THE EVOLUTION OF CORPORATE SOCIAL RESPONSIBILITY THROUGH ORGANIZATIONAL THEORIES}

\author{
José Francisco Herrera Acosta \\ Instituto Tecnológico de Sonora \\ Sonora, México \\ francisco.herrera.0869@gmail.com
}

\author{
María del Carmen Vásquez Torres \\ Instituto Tecnológico de Sonora \\ Sonora, México \\ mcvasquez@itson.edu.mx
}

\author{
Eneida Ochoa Ávila \\ Instituto Tecnológico de Sonora \\ Sonora, México \\ eneida.ochoa@itson.edu.mx
}

Fecha de recepción: 08/07/2019 - Fecha de revisión: 19/09/2019 - Fecha de aprobación: 19/12/2019 DOI: https://doi.org/10.36995/j.visiondefuturo.2020.24.02.003.es

\section{RESUMEN}

A inicios del siglo XX, se origina una de las épocas más interesantes para el ámbito organizacional, siendo reconocida esta como, la segunda revolución industrial, en donde hubo una gran cantidad de avances e innovaciones tecnológicas, cabe destacar que, dentro de esta etapa, se generaron importantes aportaciones estableciendo de esta manera las teorías organizacionales más representativas en el ámbito empresarial. De modo que, el presente documento de investigación tiene como objetivo realizar una reflexión teórica mediante una exhaustiva revisión literaria para identificar como la RSE (Responsabilidad Social Empresaria) ha sido afectada por las diversas teorías organizacionales a través de los años. En conclusión, la historia, así como los documentos que hablan acerca de las diversas teorías organizacionales, demuestran que los mayores aportes a este rubro se realizaron durante el siglo XX, en la época de la industrialización, aunque, dichos aportes estaban destinados mayormente a cuestiones de producción. Las empresas de la actualidad tienen que analizar cada uno de los beneficios que la RSE puede generar para su operación diaria, y aunque la principal barrera de que esta no sea implementada dentro del ámbito organizacional es que la mayoría de los directivos la consideran más un gasto que una inversión a largo plazo, está a ciencia cierta es una estrategia más que viable hacia el desarrollo de las organizaciones, ya que permite establecer un panorama a futuro sustentable.

PALABRAS CLAVE: Responsabilidad Social Empresarial; Organización; Teoría.

\section{ABSTRACT}

At the beginning of the twentieth century, one of the most interesting times for the organizational field originates, being recognized as the second industrial revolution, where there were a lot of advances 
and technological innovations, it should be noted that, within this stage, important contributions were generated establishing in this way the most representative organizational theories in the business field. So, this research paper aims to make a theoretical reflection through an exhaustive literary review to identify how CSR has been affected by various organizational theories over the years. In conclusion, the history, as well as the documents that talk about the various organizational theories, show that the greatest contributions to this item were made during the twentieth century, at the time of industrialization, although, these contributions were mostly aimed at issues of production. Today's companies have to analyze each of the benefits that CSR can generate for their daily operation, and although the main barrier that this is not implemented within the organizational scope is that most managers consider it more an expense that a long-term investment is for sure is a more than viable strategy towards the development of organizations, since it allows establishing a sustainable future outlook.

KEYWORDS: Corporate Social Responsibility; Organization; Theory.

\section{INTRODUCCIÓN}

A inicios del siglo XX, se origina una de las épocas más interesantes para el ámbito organizacional, siendo reconocida esta como, la segunda revolución industrial, en donde hubo una gran cantidad de avances e innovaciones tecnológicas, cabe destacar que, dentro de esta etapa, se generaron importantes aportaciones estableciendo de esta manera las teorías organizacionales más representativas en el ámbito empresarial (Ibarra y Montaño, 1987). Entre ellas está la organización científica del trabajo de Frederick Taylor (1983), caracterizada por analizar el comportamiento interno en la organizaciones, las motivaciones de los operarios, la metodología de trabajo y ocasionar resultados importantes en cuestión de productividad y eficiencia en su operación; en cambio, se desarrolla la teoría clásica de la administración de Henri Fayol (1987), considerado uno de los autores más emblemáticos en la historia administrativa, la contribución de este autor produjo diversas aportaciones para el ámbito empresarial, por ejemplo, se crearon catorce principios administrativos, se establecieron estudios técnicos con los que se determinaron las funciones del personal operativo y administrativo dentro de las unidades económicas, además se propuso la estandarización de funciones, y se plantaron las bases del proceso administrativo; otro autor a destacar durante esta época es Max Weber (1964), quien con su teoría de la burocracia se dio la tarea de comprender y analizar todos los elementos que influyen en la construcción de las estructuras sociales, en otras palabras, en comprender los procesos de los sistemas gubernamental para posteriormente implementarlos en el ámbito organizacional, cabe destacar que este autor es representativo en el área de las ciencias sociales.

Ante este contexto, las industria del siglo XX es influenciada por las aportaciones de los autores anteriormente mencionados, considerados los principales exponentes administrativos y de temas organizacionales, ya que estos, con base en sus teorías determinaron la sistematización y la estructura de las empresas, implementando un modelo de negocio y un sistema de operación capitalista, ya que 
los directivos de aquella época su principal preocupación era la de producir grandes cantidades de consumibles en el menor tiempo posible, disponiendo de forma exhaustiva del personal operativo, llamando a este fenómeno como producción en masa; se debe mencionar que, la finalidad principal de someter ante esta situación a la organización, era de vender grandes cantidades de productos a terceros, y de estos ingresos los trabajadores obtenían muy poca remuneración económica. Entonces, para responder ante esta situación, se produjeron los sindicatos con la finalidad de velar por el bienestar físico y mental del trabajador, además de luchar por sus derechos y la paga justa de su trabajo (Ibarra y Montaño, 1987).

Por otro lado, en esa época en la industria existía una gran cantidad de accidentes, muchos de estos ocasionados debido a la negligencia del trabajador, sin embargo, cualquiera que fuese el caso, la organización no respondía ante estas situaciones, por ejemplo, cuando un trabajador se accidentaba a consecuencia de cuestiones inherentes al operario, había un interés nulo del directivo por solucionar el problema, alegando que era responsabilidad única del operario el cuidar su integridad física, mental o psicológica, esto fue un detonante para la unión de los trabajadores y el sindicato, procreando de esta manera el fenómeno social reconocido como huelga (Clark, 1916). Además, no es secreto que las empresas a comienzos del siglo XX, estaban destinadas solamente para obtener ingresos y mantener un bienestar económico de los altos mandos y el desarrollo pleno de tales unidades económicas, dichos objetivos se tenían que conseguir costara lo que costara, afectando a quien se pusiera en su camino, realizando una gran cantidad de actividades poco éticas, que afectaban la salud y el bienestar de las personas que estaban ubicadas alrededor y dentro de esas organizaciones, en este caso la sociedad (Vélez, 2011).

Esto era directamente reflejando en el operador, ya que se le sometía a extensas jornadas laborales, dándole una remuneración económica bastante pobre, la vida del personal operario era despertar, saludar a su familia y trabajar, estar todo el día en la industria, produciendo una vasta cantidad de bienes, para intentar brindarle a su familia una vida digna, sin embargo, ese sudor y ese esfuerzo, todos esos resultados positivos eran hacia el directivo, ya que, estos buscaban quedarse con la mayor parte del ingreso, y era invertido en otros negocios, en tecnologías nuevas, contratar personal, comprar materia prima o gastarlo en las necesidades y deseos de los jefes (Ibarra y Montaño, 1987).

Entonces, en Europa y los Estados Unidos de Norteamérica, comienza a tomar fuerza las huelgas, la principal razón de esto, la calidad de los productos que dañaban la salud de los consumidores, el mal trato por parte de los directivos hacia los operarios, así como la mala remuneración económica y la contaminación generada por estas industrias (Carnegie, 1906). Ante este contexto, las industria del siglo $\mathrm{XX}$ es influenciada por las aportaciones de los autores anteriormente mencionados, considerados los principales exponentes administrativos y de temas organizacionales, ya que estos, con base en sus teorías determinaron la sistematización y la estructura de las empresas, implementando un modelo de negocio y un sistema de operación capitalista, ya que los directivos de aquella época su principal preocupación era la de producir grandes cantidades de consumibles en el menor tiempo posible, disponiendo de forma exhaustiva del personal operativo, llamando a este fenómeno como producción

\footnotetext{
"Visión de Futuro" Año 17, Volumen N²4 N² 2, Julio - Diciembre 2020 - Pág 82 - 104

URL de la Revista: http://visiondefuturo.fce.unam.edu.ar/index.php/visiondefuturo/index

URL del Documento: https://visiondefuturo.fce.unam.edu.ar/index.php/visiondefuturo/issue/view/18

ISSN 1668 - 8708 - Versión en Línea

E-mail: $\underline{\text { revistacientifica@fce.unam.edu.ar }}$
} 
en masa; se debe mencionar que, la finalidad principal de someter ante esta situación a la organización, era de vender grandes cantidades de productos a terceros, y de estos ingresos los trabajadores obtenían muy poca remuneración económica. Entonces, para responder ante esta situación, se produjeron los sindicatos con la finalidad de velar por el bienestar físico y mental del trabajador, además de luchar por sus derechos y la paga justa de su trabajo (Ibarra y Montaño, 1987).

Por otro lado, en esa época en la industria existía una gran cantidad de accidentes, muchos de estos ocasionados debido a la negligencia del trabajador, sin embargo, cualquiera que fuese el caso, la organización no respondía ante estas situaciones, por ejemplo, cuando un trabajador se accidentaba a consecuencia de cuestiones inherentes al operario, había un interés nulo del directivo por solucionar el problema, alegando que era responsabilidad única del operario el cuidar su integridad física, mental o psicológica, esto fue un detonante para la unión de los trabajadores y el sindicato, procreando de esta manera el fenómeno social reconocido como huelga (Clark, 1916). Además, no es secreto que las empresas a comienzos del siglo XX, estaban destinadas solamente para obtener ingresos y mantener un bienestar económico de los altos mandos y el desarrollo pleno de tales unidades económicas, dichos objetivos se tenían que conseguir costara lo que costara, afectando a quien se pusiera en su camino, realizando una gran cantidad de actividades poco éticas, que afectaban la salud y el bienestar de las personas que estaban ubicadas alrededor y dentro de esas organizaciones, en este caso la sociedad (Vélez, 2011).

Esto era directamente reflejando en el operador, ya que se le sometía a extensas jornadas laborales, dándole una remuneración económica bastante escasa, la vida del personal operario era despertar, saludar a su familia y trabajar, estar todo el día en la industria, produciendo una vasta cantidad de bienes, para intentar brindarle a su familia una vida digna, sin embargo, ese sudor y ese esfuerzo, todos esos resultados positivos eran hacia el directivo, ya que, estos buscaban quedarse con la mayor parte del ingreso, y era invertido en otros negocios, en tecnologías nuevas, contratar personal, comprar materia prima o gastarlo en las necesidades y deseos de los jefes (Ibarra y Montaño, 1987).

Entonces, en Europa y los Estados Unidos de Norteamérica, comienza a tomar fuerza las huelgas, la principal razón de esto, la calidad de los productos que dañaban la salud de los consumidores, el mal trato por parte de los directivos hacia los operarios, así como la mala rentabilidad de la organización; posterior, el directivo comienza a tomar ciertas medidas, a ser acreedor de ciertas responsabilidades ante el operario y la comunidad; surgiendo así la responsabilidad social empresarial (RSE), quien fue principalmente explicada por Bowen (1953) reconocido como padre del término. Considerando que, la RSE surge debido a las prácticas poco éticas que las empresas del siglo XX realizaban. El término es definido como, "la obligación del empresario de adoptar políticas, y desarrollar decisiones, que acompañen líneas de acción deseables según los objetivos y valores de la sociedad" (p. 6), generando una gran polémica en ese tiempo, debido a que la principal visión de las organizaciones era la de cumplir sus necesidades económicas, se dice que, en esa época, existía una, y sólo una responsabilidad: utilizar recursos y organizar actividades con el objetivo de aumentar sus ganancias.

\footnotetext{
"Visión de Futuro" Año 17, Volumen N²4 N², Julio - Diciembre 2020 - Pág 82 - 104

URL de la Revista: http://visiondefuturo.fce.unam.edu.ar/index.php/visiondefuturo/index

URL del Documento: https://visiondefuturo.fce.unam.edu.ar/index.php/visiondefuturo/issue/view/18

ISSN 1668 - 8708 - Versión en Línea

E-mail: $\underline{\text { revistacientifica@fce.unam.edu.ar }}$
} 
La obra de Bowen, tenía como fundamento principal, la percepción de los negocios, ya que, los consideraba como centros vitales, en donde el poder y la toma de decisiones, así como las actividades y acciones que realizaban, tenían una repercusión directa en la vida de las personas quienes son independientes a la actividad organizacional, afectando en muchos aspectos externos como la salud, la educación, la seguridad, entre otros (Carroll, 1999). Por lo tanto, la RSE marca un antes y un después en las empresas, teniendo un impacto directo en cómo se gestionaban éstas. Posteriormente a esto, surgen nuevas teorías, modificando de tal manera las unidades de negocio, teniendo a su vez, relación o impacto con las practicas socialmente responsables.

De modo que, el presente documento de investigación tiene como objetivo realizar una reflexión teórica mediante una exhaustiva revisión literaria para identificar como la RSE ha sido desarrollada por las diversas teorías organizacionales a través de los años. Analizando las aportaciones teóricas de autores como Kast y Rosenzweig (1988); DiMaggio y Powell (1983); Barney (1991); Evan (1967); Provan (1995); Porter (1979); Thiétart y Forgues (1995); entre otros.

\section{DESARROLLO}

\section{El Enfoque de Sistemas Organizacionales}

Todo comienza con el término de sistema, anteriormente era un concepto al cual no se le prestaba gran interés. Varios autores a través de la historia llevaron este concepto de la mano hasta darle la relevancia necesaria. No fue hasta el año de 1927 cuando Kohler encamino la teoría general de sistemas (TGS), esta aportación estaba dirigida a los aspectos generales de los sistemas inorgánicos, a diferencia de otras aportaciones las cuales estaban mayormente especializadas en sistemas orgánicos, desarrollando de esta manera la percepción de los sistemas abiertos (Bertalanffy, 1989).

Esta teoría, explica como los sistemas son considerados como un todo unitario organizado, compuesto por una o varias partes, ya sean elementos, factores, componentes o subsistemas, los cuales son interdependientes y delineados por límites que son identificables, a su supra-sistema ambiental. Cabe destacar, que el principal autor de esta teoría es Bertalanffy, y este explica como a través de los años, se desarrollaron teorías y prácticas administrativas que han cambiado las ideologías y las operaciones en las organizaciones, todo esto con base a investigaciones científicas. El enfoque de sistemas no es la excepción, ya que éste permitió integrar todas las actividades de una organización, haciéndola ver como un sistema abierto el cual mantiene una interacción con factores internos y externos, siendo integrada por diversos subsistemas (Kast \& Rosenzweig, 1988). Entonces, en la sociedad nace la necesidad del desarrollo con un enfoque basado en sistemas, debido a que se buscaba encontrar un camino en común, un medio para alcanzar determinados objetivos, tanto organizacionales como sociales (Bertalanffy, 1989).

En tal caso, es cuando comienza la relevancia del término RSE y sus elementos en el ámbito empresarial, así como su relación con la TGS, ya que, anteriormente, al considerarse a la organización como un sistema independiente y cerrado, los directivos del siglo XX, no se preocupaban por responder 
ante situaciones del contexto social, político, económico o tecnológico, solamente laboraban y se preocupaban por lo que sucedía dentro de su organización, específicamente en el área de producción y ventas. Posteriormente a las aportaciones establecidas por tal contribución, se comprende como cuestiones externas a la organización tienen una repercusión directa o indirecta en esta, un ejemplo muy claro, el trabajador al ser una fuerza interna para la operación, tiene la facultad de ejercer ciertas presiones y cuidar sus intereses. Además, en la actualidad, el enfoque de sistemas sigue vigente, ya que, las empresas tienen que responder ante las nuevas necesidades, deseos o tendencias de sus consumidores; en donde, la actual, es en un enfoque verde o sustentable, teniendo que adoptar cuestiones y elementos socialmente responsables, con la intención de seguir compitiendo en el mercado actual.

\section{La creación de necesidades sustentables}

La TGS realiza grandes cambios en el ámbito organizacional, modifica los paradigmas empresariales de gran manera, la perspectiva de operación cambia, por lo tanto, comienzan a surgir nuevas situaciones, problemáticas, deseos y necesidades en la industria. Para explicar tales necesidades, se tiene que mencionar a Abraham Maslow (1943), quien es precursor en la teoría de las nuevas relaciones humanas (TNRH), que también es considerada una escuela del pensamiento, y esta hace oposición a las aportaciones y la información relevante, generada por las teorías clásicas administrativas, siendo denominada como la Escuela del neo-humanismo y tenía como pilar fundamental, el análisis del comportamiento en los humanos, siendo objeto de estudio su motivación para la mejora de sus actividades brindando mayores índices de productividad a las organizaciones en donde desarrollaban su vida laboral; considerada una continuación a las teorías de relaciones humanas de Mayo, y una fuerte crítica a los sistemas rígidos presentados por Weber y la falta de consideración de las necesidades humanas en la teoría de la burocracia.

Maslow (1943), consideraba que existían cinco tipos de necesidades a satisfacer en los seres humanos. La primera, las necesidades fisiológicas o biológicas, esta necesidad era considerada como base para la motivación de los seres humanos, en esta, toma suma relevancia el termino de homeostasis que hace referencia a los esfuerzos del cuerpo humano por mantener un estado normal y equilibrado, ya sea por el consumo de azucares, grasa, calcio, oxigeno, por lo tanto, la respiración, el hambre y el sueño forman parte de esta necesidad; a partir de cumplir esa necesidad, se origina la segunda que era la necesidad de seguridad, entonces cualquier organismo u organización, es un mecanismo en busca de seguridad, en esta necesidad los individuos, están en busca de protección, de darle una respuesta a cosas enigmáticas y curiosas para el hombre, esta es la principal necesidad a recalcar. En tercero, al cumplir con la necesidad de alimento o seguridad, se dice que el individuo comienza a tener una nueva, la cual hace referencia a la búsqueda incesante del ser humano por pertenecer a un grupo, de dar o recibir afecto, de ser parte de una sociedad y satisfacer su necesidad de convivencia o social, siendo denominado como necesidades de pertenencia; posteriormente, la siguiente necesidad en esta jerarquía, es la necesidad de estima o reconocimiento, dentro de esta necesidad se puede observar, 
que hace suma referencia al nivel de autoestima de las personas, en esta parte, se menciona que el ser humano busca ser reconocido dentro de los grupos sociales, que busca mantener una imagen ante sus semejantes, al cumplir con esta parte surge una última necesidad; esta última es reconocida como las de autorrealización, en esta parte final, se dice que los seres humanos buscan realizar actividades que les genere estímulos positivos o de satisfacción, por último, al satisfacer todas las necesidades anteriores, el ser humano buscara la manera de realizar acciones para las que el considere fue generada su existencia.

Anteriormente se menciona la importancia de la segunda necesidad planteada por el autor, la necesidad de seguridad, esta toma una relación bastante interesante al momento de hablar de la creación y la existencia de la RSE en las organizaciones de la actualidad. Las organizaciones del siglo $\mathrm{XX}$ al operar de una manera particular y estar explotando de cierta manera a su personal, crearon condiciones ineficientes e inseguras para los trabajadores, en consecuencia, nacieron ciertas necesidades por parte de los operarios, que tuvieron que exigir por medio de los sindicatos para que estas pudieran ser escuchadas y satisfechas, ya que, en su momento los directivos ignoraban estas solicitud, y se crearon ambientes de insatisfacción laboral, entonces eso llevo a las personas que trabajan en las industrias a buscar alternativas, por eso mismo se dice que la RSE nació a partir de una necesidad, así como lo planteaba Maslow, y a consecuencia de generar tal satisfacción por medio de prácticas éticas y socialmente responsables, se originan nuevas necesidades.

\section{Un comportamiento ético}

A partir de lo planteado en la $\mathrm{TNRH}$, se comienzan a generar cambios en la industria, con base en las nuevas necesidades planteadas, entonces los directivos comienzan a tomar decisiones para producir estos cambios, a consecuencia de esto se genera una nueva teoría, que trata de explicar el complejo proceso en la toma de decisiones, conocida como la teoría del comportamiento humano (TCH). Se debe mencionar, como las organizaciones a través de los años se han visto inmersas en una gran cantidad de cambios, lo que les ha permitido evolucionar y desarrollarse en mercados cada vez más vertiginosos, aspectos como actividades, obligaciones, responsabilidades, condiciones laborales, seguridad, higiene, entre otras cosas, se han visto afectadas por los cambios ideológicos de las personas, buscando mejorar la calidad de vida y trabajo de sus participantes en busca de la productividad misma; y todo esto con base en alguna decisión tomada (March, 1988).

Por lo tanto, lo que lleva a la correcta gestión de las organizaciones es la toma de decisiones, es una facultad única que poseen los altos directivos, puede ser considerada como un proceso de reflexión y planificación la cual posteriormente, permitirá ejecutar las acciones planificadas en base a las decisiones tomadas, entonces con base en lo anterior, se puede decir existe una fuerte relación entre acción y decisión. Se cree que la toma de decisiones está enfocada al análisis de varias posibilidades o alternativas, en las cuales se realizan diferentes comparaciones y valoraciones acerca de las decisiones y consecuencias que puede traer una determinada acción, siendo vista de una perspectiva abstracta funcional (Luhmann, 1997). 
Las transacciones en los negocios de la actualidad están diseñadas para que el proceso de toma de decisiones se vea influenciado por el mercado en el que se encuentran, y estas se ven reflejadas en el desarrollo de su industria, las sociedades tienen una economía sólida y bien desarrollada, se ven envueltas en grandes organizaciones permitiendo mejoras en los sistemas sociales, económicos y organizacionales (March, 1988). Otro termino a considerar en la TCH es el concepto de racionalidad implementado por Simon (1947), el cual es considerada como un "modelo integrador en el que se amoldase el comportamiento, por medio de diversas visiones o perspectivas las cuales permiten analizar alternativas de comportamiento en diferentes situaciones" (p. 59); la reflexión acerca de cómo las decisiones y actividades pueden traer ciertos resultados; y la elección de un sistema el cual permita al individuo el regirse por ciertos valores, siendo la base de alternativas para responder ante las situaciones o problemáticas que se puedan presentar. Además, el compartimento de los individuos revela ciertos criterios de racionalidad; que el comportamiento de las personas o integrantes de una organización, genera una organización racional en cada una de sus partes, las cuales no están fuertemente interrelacionadas entre sí.

Ante este contexto, es clara la relación entre la TCH y la RSE, los empresarios comenzaron al identificar situaciones y tomar decisiones de manera racional, la problemática en aquel tiempo era bastante notable, en donde existía un descontento por parte del operario, además de la problemática en donde cuestiones ambientales estaban tomando mayor relevancia, el escenario en el que estaban inmersos les obligaba a actuar y tomar decisiones acertadas, ya que al decidir de manera espontánea y no analizar el escenario presente y futuro, podían generar situaciones en las que no se verían beneficiados, al contrario podía ocasionar un ambiente en el que estuvieran bastante vulnerables ante la competencia y por consecuencia ser superados, inclusive en el peor de los casos fracasar en el ambiente organizacional. Ahora en la actualidad, el directivo tiene la responsabilidad de tomar decisiones de manera ética, es necesario considerar cuestiones para el beneficio de la actividad empresarial, y también es una obligación del directivo el analizar posibles situaciones que puedan presentarse en el ambiente externo, entonces la RSE hace principal énfasis en eso, y se han escrito una gran cantidad de artículos haciendo referencia al liderazgo socialmente responsable, en como esa toma de decisiones es perjudicial para el desarrollo sustentable de las organizaciones y de la sociedad en cara al futuro.

\section{La contingencia responsable}

Posterior a lo planteado en la $\mathrm{TCH}$, los dirigentes en las organizaciones al tomar ciertas decisiones empezaron a obtener resultados positivos y negativos en su actividad operacional, los dirigentes más visionarios comenzaron a realizar planes permitiendo actuar ante los diferentes escenarios en los que podían estar inmersos, entonces los teóricos comenzaron a analizar esta situación y generaron otra aportación que explicaría este fenómeno en el ambiente organizacional. Titulada la teoría de la contingencia (TCON) es desarrollada a partir de los años 50's, generada a partir de investigaciones empíricas anteriormente diseñadas, las cuales tenían la finalidad de analizar y verificar las estructuras 
organizacionales de las industrias, generando nuevos conceptos y perspectivas del término, empresa. Posteriormente a estas investigaciones, se comenzó a percibir a las organizaciones como sistemas abiertos, como lo plantea la TGS, quienes se veían influenciadas por aspectos del ambiente externo e interno de las organizaciones, factores sociales, políticos o tecnológicos, empezaron a ser relevantes para la dirección de las organizaciones. En la TCON existen dos perspectivas, la de Escuelas de los sistemas Socio-técnicos en el que se, analizan los procesos y reacciones que ocurren en los ambientes estáticos y aleatorios de las organizaciones. Por otra parte, en la otra corriente, esta estudia la influencia de las contingencias sobre la estructura organizacional y el comportamiento administrativo, desarrollada por autores como Woodward, Pugh, Chandler o Crozier, quienes son los más representativos, y analizaban aspectos industriales como la producción continua, la automatización, la producción en masa, estructuras empresariales, tecnologías, entorno externo, etc. (Bueno, 1996).

Con el desarrollo de la TCON, la administración cobro gran relevancia en la historia de las grandes corporaciones, debido a las investigaciones y aportes desarrolladas por diversos autores, ya que, se dice que después de la Segunda Guerra Mundial, hubo una gran expansión de recursos económicos para el sector industrial de Estados Unidos, y por lo tanto, los directivos, se vieron obligados a especializarse en temas, aportes y teorías administrativas, sobre todo en la estructura de las organizaciones (Chandler, 1962).

Ahora bien, relacionando la TCON con la RSE, esta fue una herramienta y permitió a los altos directivos, comprender la existencia de diversos un métodos, principios universales, o teorías para la correcta gestión de todas las actividades u operaciones a realizar para la operación en la industria, o de cualquier organización, la TCON sirve como aportación orientadora, pues permite en la actualidad a los empresarios el analizar los diferentes escenarios y generar estrategias o planes que brinde opciones favorecedoras ante el entorno donde se encuentre, en otras palabras, es una herramienta para estar preparados; por ejemplo, aquellos empresarios en la época del siglo XX, que analizaron la situación de inconformidad y de abuso de recursos, crearon sus planes de contingencia para modificar su estructura y optimizar sus recursos, aquellas organizaciones que hicieron caso omiso a tal situación perdieron recursos materiales, financieros y humanos, teniendo repercusiones bastante graves en su operación, entonces se comienza a trabajar en planes de contingencia basados en aspectos éticos y sustentables, tanto para las personas como para el ambiente externo.

\section{El isomorfismo verde}

A partir de los estudios realizados con la TGS, las organizaciones comienzan a ser analizadas como un todo, en donde cuestiones internas (procesos, actividades, estructura) y externas (tecnología, política, economía) afectan de manera directa o indirecta a la operación principal. En base a lo anterior, se genera la conocida teoría institucional (TI), caracterizada por conceptos como legitimidad, isomorfismo o normas; esta teoría, se encarga de explicar cómo en las empresas, al modificar o actualizar su estructura, los procesos, las actividades o su gestión, comienza a transformarse en una institución, una organización que presenta cierta normatividad que plantea lo que es considerado

\footnotetext{
"Visión de Futuro" Año 17, Volumen N²4 N² 2, Julio - Diciembre 2020 - Pág 82 - 104

URL de la Revista: http://visiondefuturo.fce.unam.edu.ar/index.php/visiondefuturo/index

URL del Documento: https://visiondefuturo.fce.unam.edu.ar/index.php/visiondefuturo/issue/view/18

ISSN 1668 - 8708 - Versión en Línea

E-mail: $\underline{\text { revistacientifica@fce.unam.edu.ar }}$
} 
correcto o incorrecto dentro de las unidades económicas, creando condiciones así que le permiten obtener mayores probabilidades de establecerse y sobrevivir ante un mercado globalizado (Díez, Díez, y Vázquez, 2014).

Por otra parte, se crea la TI debido a la falta de orden y responsabilidades en las organizaciones del siglo XX, ya que según DiMaggio y Powell (1983) las empresas de aquella época no presentaban procesos, reglas o normas que rigieran las conductas sociales, o que permitieran realizar cursos de acción ante diversas situaciones que podían presentarse en aquel entorno. Cabe destacar que, las unidades económicas evolucionan a partir de ciertas particularidades históricas y culturales, consideradas así inmutables. La institucionalización, es percibida como un "proceso, que inicia cuando los directivos en las industrias, toman decisiones que implementen iniciativitas con la finalidad de desarrollar una gestión estratégica y cultura organizacional" (DiMaggio y Powell, 1983, p. 149); este es un proceso complejo y que requiere bastante tiempo para madurar, asentarse y ser introducido a la organización. Otro aspecto a considerar en la TI, considera un entorno cambiante, en donde aspectos internos y externos convergen, se ve en la necesidad de reestablecer sus condiciones, la principal razón de esto, son los llamados shocks exógenos, considerados como las demandas del ambiente externo; en consecuencia a lo anterior, las unidades económicas entran en un proceso de isomorfismo, entendido como un "proceso en el que obligan a las organizaciones a adaptar ciertos elementos similares al de sus competidores, con la finalidad de igualar las condiciones entre ellas" (DiMaggio y Powell, 1983, p. 149); destacando tres tipos de isomorfismo: el coercitivo, el mimético y el normativo.

Por lo tanto, se puede decir que, ante la necesidad organizacional del siglo XX, después de la revolución industrial, en donde no existía responsabilidad de la empresa hacia los consumidores y los operarios, estos comienzan a realizar demandas por medio de los sindicatos de trabajadores, para establecer nuevas y mejores condiciones hacia los operarios. Entonces, los directivos de aquella época, identificaron tal situación, ya que les estaba afectando de manera directa ante sus intereses, y toman la decisión de generar iniciativas con la intención de resolver dicho problema, creando así la RSE. Posteriormente, las demás organizaciones al identificar lo que realizó su competencia directa, y observar como tal decisión fue acertada, ya que al realizar dicha acción, de preocuparse de la calidad de sus productos, el medio ambiente y el cuidado del operario y el consumidor, resolvieron tal situación, la competencia adopta dichos elementos, normas, reglas y/o procesos establecidos por su competencia, y finalmente, entrar en un proceso de isomorfismo empresarial, estableciéndose las practicas socialmente responsables en una gran cantidad de industrias en tal solo pocos años, teniendo de esta manera una relación directa entre el isomorfismo que plantea la TI con la adopción de la RSE en los procesos organizacionales.

\section{La capacidad socialmente responsable}

Posterior a las ideas y la normatividad planteada en la TI, se genera una aportación que habla acerca de cómo las organizaciones poseen diferentes facultades y habilidades, que pueden ser utilizadas para su desarrollo y supervivencia, independientemente cual sea su giro, cantidad de 
empleados, o plataforma filosófica, las empresas poseen ciertos elementos que las hacen únicas, y que pueden jugar a favor o en contra de su desarrollo, a esta contribución se le conoce como la teoría de recursos y capacidades (TRC). Cabe destacar, el principal exponente de la TRC, es Barney (1991) quien explica que existe la posibilidad de generar una ventaja competitiva de acuerdo a las posibilidades y materiales de trabajo de cada una de las industrias existentes en el mercado, independientemente de su giro o tamaño.

Por consiguiente, la TRC hace singular relevancia a realizar análisis internos y externos dentro de las organizaciones; la principal herramienta para identificar aquellos aspectos internos y externos dentro de la organización, según lo que esta aportación propone, es por medio de una matriz FODA, ya que permite esclarecer aquellos elementos que son beneficiosos y perjudiciales de la empresas, denominados como fortalezas y debilidades, pertenecientes a factores internos; por otro lado, las amenazas y oportunidades, pertenecientes a factores externos, otro punto a recalcar, es que esta contribución plasma que con base en esta herramienta, estas unidades económicas pueden responder ante las problemáticas, los deseos y las necesidades del ambiente actual (Barney, 1991).

Ante este contexto, se identifica una teoría que explica la existencia de singularidad e individualismo en el ámbito organizacional, ya no siendo solamente considerada la organización como una unidad de negocio, sino como un organismo que posee ciertas características que le permite ser única y sobresalir en diferentes aspectos, ya sea sociales, tecnológicos, culturales, entre otros. Ahora, es notable la relación existente entre la TRC y la RSE, ya que, en la actualidad existe una gran cantidad de organizaciones privadas o del sector público y la mayoría de estas, desean obtener un distintivo o certificado que plasme la existencia de actitudes o prácticas socialmente responsables dentro de sus actividades empresariales, debido a los beneficios que estos traen tales como la mejora de la imagen corporativa, atracción de nuevos consumidores, aumento en los índices de lealtad del consumidor, entre otros. Entonces, para el logro de esto, los directivos se dan la tarea que sus unidades de negocio cumplan con ciertas normas, reglas o actividades, invirtiendo en muchas ocasiones una gran cantidad de recursos, ya sea financiero, humano o tecnológico.

Según la TRC, las unidades económicas poseen diversas metodologías y recursos para operar (Barney, 1991). Por consiguiente, el lograr el distintivo de responsabilidad social de una empresa no tendrá la misma dificultad para otra; por ejemplo, las grandes marcas que son reconocidas, al tener mayores cantidades de recurso humano, tecnológico y una fluidez de efectivo bastante regular, poseen ciertos elementos que le permitirían tener mayores probabilidades de obtener un distintivo de responsabilidad social en muy poco tiempo, en caso contrario, una de tamaño mediano o pequeño, que tiene poco en el mercado y no cuenta con una gran cantidad de personal, tardará mucho más y tendrá bastantes dificultades si quiere lograr esos objetivos, porque a diferencia de la de gran tamaño, esta puede tener un departamento encargado de alcanzar cuestiones de responsabilidad social, mientras que la otra con un personal limitado de 40 trabajadores, estará mayormente ocupada por cumplir con sus objetivos, obligaciones y responsabilidades de su puesto, que tiene que ver con la operación principal.

\footnotetext{
"Visión de Futuro" Año 17, Volumen N²4 N² 2, Julio - Diciembre 2020 - Pág 82 - 104

URL de la Revista: http://visiondefuturo.fce.unam.edu.ar/index.php/visiondefuturo/index

URL del Documento: https://visiondefuturo.fce.unam.edu.ar/index.php/visiondefuturo/issue/view/18

ISSN 1668 - 8708 - Versión en Línea

E-mail: revistacientifica@fce.unam.edu.ar
} 
En conclusión, la TRC, demuestra como las organizaciones con base en sus singularidades, puede desarrollar una ventaja competitiva sustentable, sin embargo, la competencia puede identificar esos elementos y ocasionar el fenómeno de isomorfismo, explicado con anterioridad en la primera parte del artículo, por lo tanto, en la actualidad existe una mayor cantidad de empresas socialmente responsables, con procesos estandarizados y con mayores índices de productividad, generando organizaciones más competitivas y beneficiosas para el sector público y privado, produciendo actividades que son positivas no solamente hacia la actividad empresarial, sino que también a los intereses de la sociedad, ya sea en cuestiones de salud, de medio ambiente, de educación, etc.

\section{El caos responsable}

A consecuencia de la contribución en la TRC se explican las singularidades e individualidades de las organizaciones, por tanto, surge otra aportación, una que trata de explicar cuestiones complejas, que en la actualidad ocasionan incertidumbre en el ámbito organizacional, haciendo referencia a los cambios en la operación de las empresas ya sea por la incertidumbre o al caos existente en los mercados, por cuestiones de innovación o tendencias en los diferentes ámbitos; conocida como la teoría del caos (TC), los cimientos de esta contribución comienzan a partir de la década de los sesenta (1960), fue tal su expansión, que esta comienza a ser analizada en el enfoque empresarial, considerando a los sistemas organizacionales, como sistemas caóticos (Pidal, 2009).

Cabe destacar que, según Thiétart y Forgues (1995) la palabra caos, normalmente es percibida como una "condición en donde el desorden y la confusión son predominantes" (p. 28); además, el termino es regularmente utilizado dentro del ámbito de la física-matemática, bajo la concepción de un "estado de comportamiento no predecible, que sufren los sistemas dinámicos" (p. 20). Entonces, si el caos, el desorden y la incertidumbre, pueden brindar resultados positivos o negativos hacia las organizaciones, todo depende de cómo la dirección y el personal afronte estos cambios. Aunado a que, las empresas al manejarse de manera caótica, se encuentra en un estado de crisis, y puede ser utilizada esa crisis para trascender los limites anteriormente establecidos, con la finalidad de mejorar los procesos, productos, servicios, o la estructura de la unidad de negocio.

Los cambios y avances tecnológicos son más notorios dentro de un mundo y un mercado cada vez más vertiginoso, según la TC, las personas que forman parte de estos sistemas, tienen la tarea y la obligación de adaptarse y responder ante tales situaciones (Gallardo, 2002). Ahora, relacionando la TC con la RSE; comienza una gran cantidad de caos e incertidumbre en la actividad organizacional del siglo $X X$, con la revolución industrial y los aspectos que ya fueron mencionadas a lo largo del documento, hubo resistencia a tales cambios, primero por parte del personal operativo ante las modificaciones que los directivos realizaban en las empresas, en la transformación de sistemas los organizacionales tradicionales a los de una base burocrática; además, resistencia de los directivos ante las exigencias y las alteraciones planteadas del personal operativo, para modificar tal sistema, obviamente, hubo quienes consideraron que este caos sería algo pasajero y no intervinieron. 
Sin embargo, los directivos que identificaron la situación y actuaron ante dicho caos, sufrieron grandes transformaciones que les permitió mejorar, les brindó acceso a una mayor participación en el mercado, con base en el cuidado de cuestiones laborales, ambientales, sociales; por otra parte, las organizaciones que presentaron resistencia al cambio, poco a poco fueron mermando su estructura, su operación y los empleados quienes ya estaban hartos de los malos tratos, comenzaron a emigrar a industrias que si se preocuparan por ellos, llevando a aquellas que no supieron manejar el caos, al fracaso organizacional (Carroll, 1999). En la actualidad, las empresas que no responden ante el caos del mercado o de tendencias, tienen muy bajos índices de competitividad, y por lo tanto son fácilmente superadas ante sus adversarios; hoy en día, la tendencia principal es el enfoque verde establecido debido a las practicas socialmente responsables, y esto inclusive produce caos, ya que, aquellas que no se enfoquen en el cuidado ambiental y lo consideren algo intrascendente, de igual manera se irán mermando poco a poco, tal como aquellas unidades económicas que se resistieron al caos y al cambio en el siglo XX (Gallardo, 2002).

Se debe mencionar, aquellas unidades económicas que decidan ignorar las razones para constituirse como empresas socialmente responsables llegara un punto donde su operación regular le afectará, ya que, la tendencia verde es cada vez más necesaria, los consumidores y trabajadores de la actualidad, son influenciados por cuestiones de compromiso ético y responsable a orientar su decisión de compra e inclusive de trabajo (Grupo de Investigación Expok, 2015); otro punto a tratar, es que los gobiernos cada vez son más exigentes en relación a este tema, razón por la que se han definido una serie de normativas, y no con la finalidad de obtener un distintivo de responsabilidad social, sino como elementos que tienen que cumplir las organizaciones de la actualidad con el objetivo de permanecer en el mercado, un estándar; por tal motivo, los directivos de las empresas tienen que estar al tanto, acerca de su ambiente externo, de comprender e interactuar con las tendencias de hoy en día, estar preparados para cumplir con las normas u obligaciones necesarias si pretenden operar de manera competitiva.

\section{La responsabilidad interorganizacional}

Ahora bien, la TGS sistemas de Bertalanffy (1989), determina que la organización es un sistema, el cual se encuentra posicionado sobre un supra-sistema, que además este posee ciertos subsistemas, que permiten desarrollar la operación empresarial de manera óptima. Por lo tanto, si se observa a las empresas como un sistema, están cuentan con departamentos que pueden ser considerados subsistemas que componen al sistema organizacional, sin embargo, estos sistemas se ven inmersos en un ambiente externo que es considerado un supra-sistema, un sistema mayor, el cual sería la sociedad. Ahora, al tener más clara la perspectiva de la organización en base al enfoque de sistemas, se hace principal énfasis en la siguiente teoría en el subsistema más importante que las componen, el talento humano y estos son quienes juegan diversos papeles o roles dentro de la organización, y que, a su vez, transmiten ideas, conocimientos e información por redes dentro de esta, por lo tanto, para explicar este fenómeno o situación dentro del contexto organizacional, se desarrolla la teoría de relaciones interorganizacionales (TRI), siendo sus principales precursores Evan (1967) y Provan (1995).

\footnotetext{
"Visión de Futuro" Año 17, Volumen N²4 N², Julio - Diciembre 2020 - Pág 82 - 104

URL de la Revista: http://visiondefuturo.fce.unam.edu.ar/index.php/visiondefuturo/index

URL del Documento: https://visiondefuturo.fce.unam.edu.ar/index.php/visiondefuturo/issue/view/18

ISSN 1668 - 8708 - Versión en Línea

E-mail: $\underline{\text { revistacientifica@fce.unam.edu.ar }}$
} 
Regularmente las investigaciones que tienen por objetivo estudiar las relaciones interorganizacionales, se especializan en analizar las redes de transmisión de información dentro de las empresas (Provan, 1995). Según Evan (1967), las redes son "herramientas internas, que permiten circular y transmitir información o recursos que son considerados significativos para la operación organizacional" (p. 209); además, estas redes facilitan de gran manera la participación y los intercambios de información entre individuos o departamentos, generando tales beneficios como el flujo de información y un mayor número de conexiones entre las personas, resultando así al logro de los objetivos empresariales de una manera más concreta y directa.

Por otro lado, esta misma teoría establece ciertos roles o papeles que juegan las organizaciones y los individuos en diversos escenarios, denominado este último como orbita de rol. Además, se describe una órbita organizacional, que explica acerca de la configuración de una empresa o industria, arrojando otro tipo de orbitas que determinan su estructura (Provan, 1995). Aplicando estos elementos en cuestiones de RSE, las unidades económicas se ven inmersas en una gran cantidad de actividades, operaciones y demás, normalmente, la organización está sumergida en el rol de empresa, la cual busca simplemente generar ingresos para satisfacer las necesidades de los integrantes de esta; sin embargo en la actualidad, se establece otro rol, el de empresa socialmente responsable, y la principal característica de este es el desarrollo de prácticas e iniciativas que cuiden cuestiones ambientales, sociales, culturales, entre otros.

Entonces se habla de cómo las organizaciones pueden jugar distintos roles dentro de la sociedad, así como sus trabajadores, por ejemplo, se establece a los empleados como jugadores de un rol operario dentro de una organización, además de jugar el rol de promotores de la salud, cuidadores ambientales, de seguridad, son temas de RSE, y en cambio la órbita organizacional es caracterizada por las empresas, así como su estructura; además, las organizaciones de referencia, que en este caso serían la competencia directa, y el desarrollo de actividades socialmente responsables.

\section{Una estrategia verde}

Posterior a establecer las redes interorganziacionales, el término de estrategia comienza a captar el interés de los directivos, regularmente este concepto es relacionado con el autor Michael Porter (1981), este sin dar lugar a dudas es uno de los más emblemáticos en el rubro, y la define "la manera en cómo las organizaciones compiten ante su entorno, tomando elementos como objetivos, productos, mercado, mercadotecnia, fabricación, haciéndole frente a las situaciones que se puedan presentar en este" ( $p, 610)$. La estrategia es considerada una herramienta para las empresas de innovación en cuestión de producto, servicio e inclusive lograr un mayor posicionamiento en el mercado, donde se ve inmersa la actividad empresarial, por otro lado, la estrategia toma singular relevancia en el ámbito administrativo y organizacional, tanto que ha pasado a formar parte del proceso administrativo implementado por Fayol (1987), además, la esencia principal de la estrategia, es perteneciente al proceso de toma de decisiones; permitiendo a las unidades económicas desarrollar objetivos que establezcan mayores índices de competitividad para la actividad empresarial. 
Por otra parte, Porter (1979) plantea cinco fuerzas dominantes en la implementación de la estrategia dentro de la organización, las cuales son: la amenaza de entrada de nuevos competidores; el poder de los proveedores; el poder de los compradores; la amenaza de los substitutos; y la rivalidad entre competidores. Cabe destacar, que quienes tienen la mayor responsabilidad, al momento de crear, desarrollar e implementar una estrategia, así como la correcta gestión de las organizaciones, son los altos directivos, quienes, en los últimos años, han tomado ciertas decisiones, que perjudicaron la imagen corporativa de estas, teniendo repercusión directa en su rentabilidad y su competitividad en el mercado (Pless, 2007). Tal es el caso, de una empresa automotriz reconocida a escala mundial; el CEO, quien estaba a cargo de esta, tomo la decisión de utilizar una estrategia poco ética, ya que modifica de tal manera más de once millones de unidades, para que los tableros de control especificaran una baja emisión de gases contaminantes; estos fueron detectados por un tercero y en consecuencia la empresa pierde el $40 \%$ de su valor en el mercado, teniendo la dirección que renunciar, debido a que se optó por una decisión poco ética (Solís, 2015).

En la actualidad, quienes dirigen a las organizaciones, son conscientes acerca de cómo sus decisiones pueden tener repercusiones positivas o negativas en diversos rubros, ya sea la salud, la seguridad, el ambiente, entre otros; por eso mismo, los directivos están mayormente preocupados por generar estrategias que tengan relación con temas de sustentabilidad, y que estén encadenadas a actividades socialmente responsables, con la finalidad de generar valor ante las personas, y realizar actividades para apoyar a la situación actual en el mundo. Una estrategia erróneamente implementada, o decisiones que no estén enfocadas en cuestiones verdes, pueden ocasionar el fracaso en la organización, o la salida del directivo, esto debido a la falta de una percepción verde (Pless, 2007).

\section{El desarrollo socialmente responsable}

Después de hablar acerca de la estrategia, se debe mencionar como a lo largo de la historia, el área empresarial ha recibido una gran cantidad de cambios y modificaciones, diversos pensadores han aportado una gran cantidad de teorías e información, que permitieron el mejoramiento de estructura, procesos y operación de las industrias, el gobierno, siendo el principal recurso para lograr esto, el capital humano. Ante esta situación, se genera una teoría que busca explicar la necesidad de mejora y el cómo hacerlo en las empresas, reconocida como la teoría del desarrollo organizacional (TDO), teniendo repercusiones en cuestiones de innovación basado en un enfoque de mejora continua (Zapata, 2007).

Entonces, la TDO es generada debido a una falta de capacidad administrativa tanto en el área de dirección como de gestión, ya que, se identifica la necesidad de modificar las estructuras y los sistemas institucionales con la finalidad de alcanzar un desarrollo económico enfocado en estrategias que permitieran el pleno crecimiento organizacional de las empresas (Bolaños, 2011). Otro aspecto importante del que habla esta aportación, es que las organizaciones viven en un entorno cambiante, por lo tanto, estas deben presentar estructuras flexibles, en donde, rápidamente puedan responder ante las nuevas tendencias y exigencias del mercado, con la finalidad de aumentar su capacidad y

\footnotetext{
"Visión de Futuro" Año 17, Volumen N²4 N², Julio - Diciembre 2020 - Pág 82 - 104

URL de la Revista: http://visiondefuturo.fce.unam.edu.ar/index.php/visiondefuturo/index

URL del Documento: https://visiondefuturo.fce.unam.edu.ar/index.php/visiondefuturo/issue/view/18

ISSN 1668 - 8708 - Versión en Línea

E-mail: $\underline{\text { revistacientifica@fce.unam.edu.ar }}$
} 
competitividad, rompiendo los paradigmas empresariales tradicionales, impulsando iniciativas para el desarrollo de nuevas y mejores capacidades (Molina, 2000).

Ahora, relacionando la TDO con la RSE, actualmente hay una tendencia por la sustentabilidad y el cuidado ambiental, se considera una realidad apremiante que existe un compromiso ético y socialmente responsable por parte de las empresas, y no solo la existencia de isomorfismo entre organizaciones, sino que gobiernos y consumidores exigen esta actividad, hoy en día, esto es un determinante y en muchas ocasiones una cuestión definitiva para adquirir un producto o un servicio. Uno de los aspectos importantes dentro de esta teoría, es que el fomentar el desarrollo dentro de la organización parte de tres constructos, el liderazgo, el aprendizaje organizacional y la cultura organizacional; curiosamente, estos tres elementos están directamente relacionados con la RSE primeramente se tiene que aclarar aspectos intervienen y que actividades son desarrolladas dentro de este rubro, la manera en cómo se realizan y los beneficios que generan; en otras palabras, se debe generar un conocimiento previo acerca de esta índole si se quiere implementar dentro de las unidades económicas; un punto a considerar es que se debe fomentar una cultura socialmente responsable en el desarrollo de las actividades empresariales, partiendo desde la dirección, siempre en beneficio de cuestiones ambientales, sociales, culturales, los directivos deben cuidar el desarrollo de sus actividades, hasta el más mínimo detalle, para que los mandos medios y operativos puedan entender la importancia de este concepto, no solo dentro de la organización, sino también fuera, en la sociedad.

Finalmente Pless (2007), introduce el término de liderazgo socialmente responsable, de aquí parten una gran cantidad de actividades y beneficios; se establece ese tipo de liderazgo, debido a que en años anteriores, surgieron una gran cantidad de escándalos, situaciones y problemáticas en el ámbito empresarial, y no provenientes de la operación de la organización, sino por decisiones de los directivos, decisiones poco éticas y que afectaron de cierta manera cuestiones de seguridad, ambiente o culturales de la sociedad. Al establecer este tipo de liderazgo socialmente responsable ocasionara un desarrollo sostenible a las organizaciones, y por lo tanto traería ciertos beneficios a las empresas y la sociedad, el más notable, sería que principios o cuestiones socialmente responsables serian transmitidas de manera mucho más sencilla, y llegarían a tener mayor relevancia y tendría resultados de mayor impacto para la sociedad; entonces, los directivos tienen la responsabilidad de cuidar y fomentar estas actividades por medio de su liderazgo. Si esto se realiza correctamente, la organización mejoraría, no solo en su estructura, también en sus procesos y en su plataforma filosófica, condiciones que son primordiales en la TDO (Molina, 2000).

\section{La tendencia verde}

A causa de lo propuesto en la TDO se comienzan a generar una gran cantidad de ideas, de prácticas, de nuevas tendencias; actualmente, existen una gran cantidad de temas que son de suma relevancia para los altos directivos, entre ellas la reingeniería de procesos, el benchmarking, el outsourcing, el empowerment, entre otros. Sin embargo, se tiene que destacar en esta parte, la sustentabilidad y la RSE.

\footnotetext{
"Visión de Futuro" Año 17, Volumen N²4 N², Julio - Diciembre 2020 - Pág 82 - 104

URL de la Revista: http://visiondefuturo.fce.unam.edu.ar/index.php/visiondefuturo/index

URL del Documento: https://visiondefuturo.fce.unam.edu.ar/index.php/visiondefuturo/issue/view/18

ISSN 1668 - 8708 - Versión en Línea

E-mail: $\underline{\text { revistacientifica@fce.unam.edu.ar }}$
} 
Hoy en día, se vive un crecimiento exponencial de organizaciones y de personas, mientras más crecen estos dos elementos es mayor la necesidad por consumir recursos naturales y la generación de residuos contaminantes aumenta, teniendo un impacto negativo desarrollando de esta manera conflictos ecológicos (Martinez, 2004). Ante esta situación, se crea una tendencia entre los consumidores, los trabajadores y los directivos, por empezar a cuidar estos aspectos, ya que los niveles de contaminación alrededor del mundo son bastante altos, y como respuesta a tal situación se han buscado identificar a los sujetos que poseen los recursos y las capacidades necesarias para solucionar la problemática, entre ellos se encuentran las unidades económicas, ya que estas poseen los ingresos y el capital necesario que permite desarrollar actividades e iniciativas, de índole sustentable.

Es necesario señalar, como la RSE, es una tendencia a nivel nacional y también mundial; por ejemplo, un grupo de investigadores denominados Grupo de Gestión Expok (2015) realizaron una investigación referente a temas socialmente responsables, demostrando como en una muestra representativa, el 98\% conocía el término, además de identificar su logo correspondiente en México; por otro lado, los entrevistados reconocieron la importancia de la RSE en la actualidad, de los distintivos que se les otorga a las empresas que desarrollan dichas actividades y expresaron la necesidad de brindarles reconocimientos a estas organizaciones por sus actividades realizadas. Posterior, en esta misma investigación, se habla acerca de cómo el consumidor está dispuesto a pagar un poco más de lo acostumbrado, si está seguro que los productos o servicios ofrecidos, provienen de una empresa socialmente responsable; posterior a esto, se identifica como la ética existente en las unidades económicas son una determinante para la decisión de compra, inclusive del trabajo de las personas y su reputación.

Asimismo, esta tendencia verde surge debido a la situación actual que se vive, según la Organización Mundial de la Salud (2018), el 92\% de las personas en el mundo es perjudicada en su salud debido a cuestiones ambientales, causando de esta manera casi el $12 \%$ de muertes alrededor del mundo por problemas respiratorios, a consecuencia de gases que son provenientes de la operación industrial, causando de esta manera más de 6.5 millones de muertes al año. Entonces, ante este tipo de situaciones, las personas, ya sea consumidores, empleados, directivos o simplemente individuos, tienden a tener una mayor preocupación por las condiciones ambientales, por adquirir ciertos compromisos y obligaciones éticas con la sociedad que les permita mejorar su calidad de vida.

Cabe destacar, que no solamente el cuidado del aire se ha vuelto una tendencia, también el cuidado y ahorro del agua, la limpieza de las calles, el evitar químicos o fertilizantes que sean dañinos para la salud, actualmente, es una responsabilidad tanto de las personas como de las organizaciones el cuidado de la sociedad, finalmente, se debe mencionar, que es de suma importancia las actividades y las decisiones que tomen los dirigentes de las sociedades y de las empresas, ya que, con base a esto, se plantearán los escenarios futuros de las próximas generaciones, que en muchos casos puede ser beneficioso o perjudicial para la población en general. 


\section{CONCLUSIÓN}

En conclusión, la historia, así como los documentos que hablan acerca de las diversas teorías organizacionales, demuestran que los mayores aportes a este rubro se realizaron durante el siglo XX, en la época de la industrialización, aunque, dichos aportes estaban destinados mayormente a cuestiones de producción.

Antes de la revolución industrial, en las organizaciones se trabajaba de una manera más flexible, el empleado aparentemente tenía mayores libertades, ya que su vida no estaba destinada solamente para la organización, posterior a las primeras aportaciones de Taylor, Weber y Fayol, esto cambia de manera radical, los directivos comienzan a adoptar ciertos sistemas más rígidos, que abusaban de la autoridad y de la jerarquía, generando así el descontento y muchas quejas por parte del personal operativo, dando pie al surgimiento de nuevas necesidades y tendencias en el ámbito organizacional.

A lo largo de la historia se han realizado diversas críticas acerca del sistema capitalista anterior y actual, era un completo abuso hacia el operario, otra cuestión a tratar, eran los malos tratos por parte de los altos y medios mandos hacia el subordinado; los jefes o supervisores comienzan a ser llamados burócratas, teniendo actitudes deplorables hacia los puestos bajos, exigiéndoles una gran cantidad de cosas, como si estos fueran máquinas, como si estos fueran de su propiedad, tal fenómeno es explicado de manera más detallada en la TI, en donde se habla acerca de cómo el ser humano, nace, vive y muere siendo parte de una organización. Pasan bastantes años de no esclavitud, sino de abusos por parte de los altos mandos además de que al querer optimizar recursos comienzan a realizar actividades poco éticas que tendrían resultados negativos a largo plazo, la compra de materia prima más económica y de menor calidad por los componentes con los que fueron formados dañarían la salud de los consumidores y los trabajadores.

Entonces, comienzan las primeras huelgas, un fenómeno social y extraordinario, la gente inconforme por el trato o ser considerada una herramienta se une a los sindicatos que serían considerados la salvación para el operario, serían la llave o herramienta que abriría nuevos senderos, ya que, permitiría la negociación de las problemáticas y situaciones de incertidumbre en la organización, y es curioso, ya que en el siglo XX existieron jefes, supervisores, consejos directivos, burócratas, entre otras formas de referirse a quien se le asignaba la responsabilidad de administrar los recursos de las organizaciones, éstos identificaron la situación, se dieron cuenta que ya era hora de adquirir ciertas responsabilidades, ya no solamente en aspectos administrativas u operativas, sino que más humanas. Las empresas comienzan a modificar su estructura, comienzan a preocuparse de la salud mental y física del trabajador, tratan de eliminar la sistematización o mecanización, las jornadas laborales son menores, se les otorga a los operarios espacios de descanso, permiten el desarrollo de grupos informales de trabajo, la industria comienza a responder por los accidentes en los espacios laborales, los productos aumentan de calidad; es decir, se empieza a promover por el bienestar del personal y del consumidor.

Nace la RSE, y hubo organizaciones que resistieron al cambio, hubo personas que tomaron la decisión de no seguir la tendencia del bienestar por el operario y el consumidor, dejando una estructura

\footnotetext{
"Visión de Futuro" Año 17, Volumen N²4 N², Julio - Diciembre 2020 - Pág 82 - 104

URL de la Revista: http://visiondefuturo.fce.unam.edu.ar/index.php/visiondefuturo/index

URL del Documento: https://visiondefuturo.fce.unam.edu.ar/index.php/visiondefuturo/issue/view/18

ISSN 1668 - 8708 - Versión en Línea

E-mail: $\underline{\text { revistacientifica@fce.unam.edu.ar }}$
} 
rígida, teniendo resultados bastante negativos para esas unidades económicas. Los demás operarios, al percatarse de lo que pasaba en otras empresas de identificar que se les daba un mejor trato a los demás, comienzan a realizar las mismas acciones, a exigir, y al no llegar a una negociación concreta con el directivo, emigran a otra; considerado el recurso más importante de su desarrollo, el talento humano, y estas llegan al fracaso mientras que las demás que decidieron implementar un enfoque socialmente responsable, aumentaron sus recursos, por lo tanto, aumentan sus ingresos y el desarrollo organizacional de las demás unidades económicas comienza.

Los indicios o necesidades de RSE surgen con base en las deficientes condiciones de las empresas, ya que, al estar en constante búsqueda de producir bienes y servicios, por generar ganancias, establecen el fenómeno de producción en masa. La industria comienza a consumir gran cantidad de recursos, ya sea financieros, tecnológicos y especialmente naturales, los productores empiezan a adquirir grandes cantidades de madera, de carbón, de plástico para producir sus artículos, y al consumir energía eléctrica y demás, produjeron vastas cantidades de $\mathrm{CO}^{2}$, los índices de contaminación aumentaron de forma alarmante, entonces el consumidor identifica tal situación, se percata que por la actividad operacional, no solo la región, sino que el mundo es afectado, por lo tanto, los compradores comienzan a adquirir productos y servicios que dañen muy poco o nada al medio ambiente de empresas en las que ellos percibían que no estaban fomentando el daño a la sociedad, teniendo un nuevo enfoque socialmente responsable, el cuidado ambiental que sería este el principal durante muchos años.

Hoy en día, inclusive al identificar como es la situación actual en cuestiones de sustentabilidad existen organizaciones que se resisten al cambio, que pretenden seguir operando de la misma manera, en donde no realizan actividades o prácticas que fomenten la responsabilidad social, teniendo la mentalidad de los directivos del siglo XX, la de solamente establecer una unidad económica para generar ganancias, en vez de fomentar un pensamiento o una cultura socialmente responsable que permita desarrollar actividades que beneficien a la sociedad, por lo tanto, al no realizar este tipo de actividades o considerar estas prácticas, dichas empresas tienen repercusiones negativas en sus ingresos y la sociedad, mientras que aquellas que responden ante la nueva tendencia se ven beneficiadas por tal situación.

En la actualidad, por medio de las redes sociales, las personas se pueden informar acerca un millón de cosas, las noticias en la red están en cuestión de segundos ya sea en las computadoras o los Smartphone de las personas, el ser humano está al tanto de cómo está la situación en niveles de contaminación, y ya no solo por medio de estadísticas, ya que, existe una gran cantidad de información, de fotos, de videos, pruebas contundentes de como la industria y los seres humanos, han tomado decisiones erróneas o poco éticas, abusando así de los recursos naturales y teniendo repercusiones bastante graves, siendo la principal problemática en la actualidad. De igual manera, es curioso, como a lo largo de los años por medio de anuncios en el periódico, spots publicitarios, artículos de revistas, e inclusive documentales que hablan acerca de la actualidad ambiental, el ser humano permanece ignorante ante tal situación, o no le toma gran importancia, inclusive con las evidencias que pueden ser analizadas todos los días y verificar los cambios de este, hay líderes mundiales que creen que el

\footnotetext{
"Visión de Futuro" Año 17, Volumen N²4 N² 2, Julio - Diciembre 2020 - Pág 82 - 104

URL de la Revista: http://visiondefuturo.fce.unam.edu.ar/index.php/visiondefuturo/index

URL del Documento: https://visiondefuturo.fce.unam.edu.ar/index.php/visiondefuturo/issue/view/18

ISSN 1668 - 8708 - Versión en Línea

E-mail: revistacientifica@fce.unam.edu.ar
} 
calentamiento global y los altos niveles de contaminación proveniente de las empresas es una mentira, claro está, que quieren proteger sus propios intereses económicos.

Los medios de información plasman que existe una crisis ya no solo en cuestiones ambientales, sino que en sociales, educativas, de salud, de seguridad, o económicas, proponiendo una gran cantidad de programas y diversas soluciones bastante complejas para erradicar esta situación, se generan propuestas rebuscadas y los dirigentes de las naciones no se percatan que las empresas son la respuesta a todas estas condiciones, cada una tiene sus diferentes particularidades, así como la expresa la TRC, y cada una de estas desarrolla un enfoque socialmente responsable a su manera, con base a lo que se tiene a la mano.

La RSE, no puede y no debe ser tomada a la ligera, ya que es una herramienta de desarrollo social bastante poderosa, ya no solo es cuidar indicadores de medio ambiente, aunque sea el punto más importante en la actualidad a escala mundial, ya que mejora cuestiones internas de una sociedad, otro punto a tratar es que la responsabilidad social no es solo tarea de las empresas o la industria, sino que en otras organizaciones de índole no lucrativas, como hospitales, iglesias, comunidades e inclusive los hogares pueden desarrollar iniciativas que promuevan el cuidado de estos aspectos, existe una gran cantidad de actividades que individualmente se pueden realizar, con la intención de mejorar la sociedad, pero esto es algo que todas las personas tienen que apoyar, no solo unas cuantas; crease o no, las organizaciones de índole empresarial tienen la facultad de impulsar a la sociedad, esto debido a que tienen los recursos necesarios para generar un impacto significativo.

La participación del ser humano es crucial para la situación actual en el mundo, las decisiones que tomen las generaciones actuales son determinantes, ya que establecerán el panorama a futuro en los años siguientes, por lo tanto, se debe tener muy en claro, que cuestiones éticas y un liderazgo socialmente responsable, puede ser beneficioso no solamente si se quieren lograr los objetivos empresariales, o tener una mayor participación en el mercado o mayor competitividad, es importante también enfocarse en los aspectos más humanos y no únicamente en los relacionados con el mercado y la competencia.

En otra perspectiva, las empresas de la actualidad tienen que analizar cada uno de los beneficios que la RSE puede generar para su operación diaria, y aunque la principal barrera de que esta no sea implementada dentro del ámbito organizacional es que la mayoría de los directivos la consideran más un gasto que una inversión a largo plazo, está a ciencia cierta es una estrategia más que viable hacia el desarrollo de las organizaciones, ya que permite establecer un panorama a futuro sustentable, creando planes que tengan que ver con las directrices o practicas socialmente responsables, además de analizar las futuras tendencias y establecer planes de acción para los escenarios futuros. Por otra parte, la RSE no solamente tiene que ser implementada dentro del modelo de negocio de las unidades económicas como algo ambiguo, que solo es utilizado el termino como una limpiadora de la imagen corporativa o algo que solamente que las obliga a realizar limpiezas de manera periódica en las calles de las ciudades, sino que tiene que ser un término que sea implementado desde la raíz de la organización, que sea parte de su cultura, que día con día se trabaje de tal manera que el enfoque de sustentabilidad este 
estrictamente implementado en las actividades desde los mandos bajos hasta los mandos altos, e inclusive este tipo de cuestiones tiene que ser fomentada por el mando alto, este tiene que poner el ejemplo, ese liderazgo socialmente responsable, y que sea considerado el enfoque sustentable como no algo extra, sino parte de la esencia organización.

Otro punto a destacar, es como las grandes marcas han sido las principales exponentes a la hora de hablar de cuestiones socialmente responsables, ya que estas organizaciones, al tener mayores recursos e ingresos que las de dimensiones pequeñas o medianas, son influyentes o determinantes al momento de hablar de temas de sustentabilidad, pero se debe analizar que, es cierto que estas poseen mayores recursos para generar este tipo de actividades, pero las empresas de índole mediana o pequeña son en mayor cantidad que las grandes, por lo tanto, si las personas que dirigen las pequeñas o medianas comenzaran a realizar actividades o reunirse a compartir ideas, crear proyectos, de seguro tendrían efectos más trascendentes que las grandes marcas, esto debido a que la cantidad de empresas menores es mayor, y por lo tanto, poseerían en conjunto mayores posibilidades, recursos y capacidades para desarrollar actividades que tengan un resultado contundente para la sociedad.

Pero, también está la problemática que, si los empresarios comienzan a percibir a la RSE como un gasto y no una inversión, esto debido a que normalmente los dirigentes de medianas o pequeñas organizaciones buscan generar resultados de manera rápida, esta puede entrar en crisis y dejar de ser considerada como una herramienta para el desarrollo sustentable de la sociedad. Puede llegar el punto en que las practicas socialmente responsables sean consideradas una tendencia no sostenible la cual este destinada a desaparecer, esto debido a que las empresas en los últimos años no han adaptado la RSE de manera concreta dentro de su cultura organizacional, sino que la han adoptado de un modo superficial, en el cual, solamente buscan obtener el distintivo socialmente responsable, y por lo tanto, es necesario el institucionalizarla ya que esto permitirá vincular las practicas socialmente responsables en la cultura empresarial. Entonces, es necesario modificar todo lo referente estas prácticas y dejar de percibirla como, el cumplir una serie de requerimientos y obtener un distintivo, es necesario el generar un modelo o una normatividad que permita gestionar de manera eficiente estas prácticas, y que puedan estar presentes desde la plataforma filosófica, que las estrategias, la misión y las actividades dentro de la organización estén vinculadas con el desarrollo y la aportación a la sociedad, por medio de una cultura socialmente responsable.

Finalmente, se debe mencionar que en los últimos años se han generado una gran cantidad de investigaciones referente a temas de sustentabilidad o RSE, sin embargo, este tipo de investigaciones son necesarias, ya que cada vez se hacen nuevos descubrimientos que permiten a las organizaciones el adoptar nuevas tendencias, que les permite implementar dentro de sus modelos de negocio y obtienen mejores resultados, obtienen un mayor alcance que les permite no solamente tener un distintivo en muchos de los casos, sino que producir resultados que son beneficiosos hacia la sociedad, por ejemplo, mejorar las estructuras de las instituciones educativas, aumentar las unidades de seguridad en las regiones, disminuir índices de contaminación, entre otros; la RSE es necesaria para el desarrollo sustentable no solo de las empresas, también de la humanidad.

\footnotetext{
"Visión de Futuro" Año 17, Volumen N²4 N², Julio - Diciembre 2020 - Pág 82 - 104

URL de la Revista: http://visiondefuturo.fce.unam.edu.ar/index.php/visiondefuturo/index

URL del Documento: https://visiondefuturo.fce.unam.edu.ar/index.php/visiondefuturo/issue/view/18

ISSN 1668 - 8708 - Versión en Línea

E-mail: revistacientifica@fce.unam.edu.ar
} 


\section{REFERENCIAS}

Barney, J. (1991). Film Resources and Sustained Competitive Advantage. Journal of Management, 17(1), 99-120. DOI: 10.1177/014920639101700108

Bertalanffy, L. (1989). Teoría General de los Sistemas; fundamentos, desarrollo, aplicaciones (Séptima ed.). México, D.F.: Fondo de Cultura Económica.

Bolaños, R. (2011). El desarrollo organizacional como estrategia para la modernización en la administración pública. Revista Nacional de Administración, 2(1), 135-144. Recuperado de https://dialnet.unirioja.es/servlet/articulo?codigo $=4716395$

Bowen, H. R. (1953). Social Responsibilities of the Businessman. United States, New York: Harper \& Row.

Bueno, E. (1996). Organización de empresas: estructura, procesos y modelos (segunda edición). España, Madrid: Ediciones Pirámide.

Carnegie, A. (1906). The Gospel of Wealth. North American Review, 183(599), 526-537. Recuperado de: https://www.jstor.org/stable/25105641?seq=1\#metadata info tab contents

Carroll, A. B. (1999). Corporate Social Responsibility; Evolution of a Definitional Construct. Business \& Society, 38(3), 268-295. DOI: 10.4135/9781452243986.n1

Chandler, A. D. (1962). Strategy and Structure; Chapters in the History of the Industrial Enterprise. United States, Massachusetts: The M.I.T. Press.

Clark, J. M. (1916). The Changing Basis of Economic Responsibility. Journal of Political Economy, 24(3), 209-229.

Recuperado

de

https://www.jstor.org/stable/1819437?seq=11\#metadata info tab contents

Díez, E., Díez, F., \& Vázquez, A. (2014). Antecedentes de la institucionalización en las organizaciones. Cuadernos de Gestión, 15(1), 15-38. DOI: 10.5295/cdg.130416ed

DiMaggio, P. J., \& Powell, W. W. (1983). The Iron Cage Revisited: Institutional Isomorphism and Collective Rationality in Organizational Fields. American Sociological Review, 48, 147-160. DOI: $10.2307 / 2095101$

Evan, W. M. (1967). La Orbita de la Organización: Hacia una Teoría de las Relaciones Interorganizacionales. Argentina, Buenos Aires: OMEBA.

Fayol, H. (1987). Administración Industrial y General; Coordinación-Control-Previsión-OrganizaciónMando (Décimo cuarta ed.). Argentina, Buenos Aires: El Ateneo.

Gallardo, A. (2002). La Era de la incertidumbre, la organización y la teoría del caos. Administración y Organizaciones, 4(8), 63-76. Recuperado de https://biblat.unam.mx/es/revista/administraciony-organizaciones/articulo/la-era-de-la-incertidumbre-la-organizacion-y-la-teoria-del-caos

Grupo de Investigación Expok. (2015). Estudio de Responsabilidad Social Empresarial en México. Recuperado de Expok: https://www.expoknews.com/estudio-sobre-responsabilidad-socialempresarial-en-mexico-expok-2015/

Ibarra, E., \& Montaño, L. (1987). Mito y poder en las organizaciones; Un análisis crítico de la teoría de la organización (Segunda ed.). México, D.F.: Editorial Trillas.

Kast, F. E., \& Rosenzweig, J. E. (1988). Administración en las organizaciones; enfoque de sistemas y de contingencias (Cuarta ed.). México, D.F.: McGraw-Hill.

Luhmann, N. (1997). Organización y decisión. Autopoiesis, acción y entendimiento comunicativo (Primera ed.). Chile: Anthropos.

March, J. (1988). Decisions and Organizations. Basil Blackwell.

Martinez, J. (2004). Los conflictos ecológico-distributivos y los indicadores de sustentabilidad. Revista Iberoamericana de Economía y Ecológica, 1, 21-30.

Maslow, A. H. (1943). A Theory of Human Motivation. Psychological Review, 50(4), 370-396.

\footnotetext{
"Visión de Futuro" Año 17, Volumen N²4 N², Julio - Diciembre 2020 - Pág 82 - 104

URL de la Revista: http://visiondefuturo.fce.unam.edu.ar/index.php/visiondefuturo/index

URL del Documento: https://visiondefuturo.fce.unam.edu.ar/index.php/visiondefuturo/issue/view/18

ISSN 1668 - 8708 - Versión en Línea

E-mail: revistacientifica@fce.unam.edu.ar
} 
Molina, H. (2000). El desarrollo organizacional como facilitador del cambio. Estudios Gerenciales, 16(77), 13-25. Recuperado de http://www.scielo.org.co/scielo.php?script=sci arttext\&pid=S0123-59232000000400001

Organización Mundial de la Salud. (2018). Air pollution. Recuperado de: https://www.who.int/airpollution/en/

Pidal, M. J. (2009). La Teoría del Caos en las Organizaciones. Cuadernos Unimetanos, 18, 29-33. Recuperado de https// Dialnet-LaTeoriaDelCaosEnLasOrganizaciones-3998894\%20(1).pdf

Pless, N. (2007). Understanding Responsible Leadership: Role Identity and Motivational Drivers. Journal of Business Ethics, 74(4), 437-456. DOI: 10.1007/sI0551-007-9518-x

Porter, M. E. (1979). How competitive forces shape strategy. Harvard Business Review, 57, 137-145. Recuperado de https://hbr.org/1979/03/how-competitive-forces-shape-strategy

Porter, M. E. (1981). The Contributions of Industrial Organization To Strategic Management. Academy of Management Review, 6(4), 609-620. DOI: 10.2307/257639

Provan, K. G. (1995). A Preliminary Theory of Interorganizational Network Effectiveness: A Comparative Study of Four Community Mental Health Systems. Administrative Science Quarterly, 40(1), 133. DOI: $10.2307 / 2393698$

Simon, H. (1947). El comportamiento administrativo; Estudio de los procesos de adopción de decisiones en la organización administrativa. México, D.F.: Aguilar.

Solís, A. (2015). 4 escándalos corporativos del 2015. Recuperado de https://www.forbes.com.mx/4escandalos-corporativos-de-2015/

Taylor, F. W. (1983). Principios de la Administración Científica (Vigésima séptima al español ed.). México, D.F.: Herrero Hermanos, Sucs., S.A. México.

Thiétart, R. A., \& Forgues, B. (1995). Chaos Theory and Organization. Organization Science, 6(1), 1931. DOI: $10.1287 /$ orsc.6.1.19

Vélez, E. (2011). Un recorrido hacia la Responsabilidad Social Corporativa. Revista Ciencias Estratégicas, 19(25), 55-74. Recuperado de http://www.redalyc.org/pdf/1513/151322413004.pdf

Weber, M. (1964). Economía y sociedad. México, D.F.: Fondo de Cultura Económica.

Zapata, J. C. (2007). Caracterización del desarrollo humano, el crecimiento y el desarrollo organizacional en ocho compañías prestadoras privadas de servicios en salud en la ciudad de Medellín. Revista Ciencias Estratégicas, 15(8), 211-222. Recuperado de http://www.redalyc.org/articulo.oa?id=151320354006

\section{REFERENCIAS BIOGRÁFICAS}

\section{Herrera Acosta José Francisco}

Egresado de la carrera de licenciado en administración por el Instituto Tecnológico de Sonora con vasta experiencia en el área de consultoría en la región de Sonora; actualmente estudiante de posgrado en la Maestría de Gestión Organizacional, interesado en investigaciones de responsabilidad social empresarial.

\section{Vásquez Torres María del Carmen}

Doctora en Planeación Estratégica para la Mejora del Desempeño; además de desempeñarse como profesora investigadora del Departamento de Ciencias Administrativas y Coordinador del Programa Maestría en Administración y Desarrollo de Negocios en el Instituto Tecnológico de Sonora, Cajeme, Sonora, México.

\section{Ochoa Ávila Eneida}

PhD con especialidad en administración de negocios. Doctoranda en Planeación Estratégica y Mejora de las Organizaciones. Maestra en Administración con Especialidad en Recursos Humanos y licenciada en Psicología. Profesora Titular "C", del Departamento de Psicología del Instituto Tecnológico de Sonora. Pertenece al Sistema Nacional de Investigadores Nivel I. 